\title{
ELETRENCEFALOGRAMA E DROGAS ANTICONCEPCIONAIS
}

\author{
José Geraldo Camargo lima * \\ rosa Helena longo * \\ Waldemar D. Pereira de Carvalho ** \\ Helio Brito Di Migueli ***
}

Mediante experimentação em animais têm sido assinaladas alterações eletrencefalográficas atribuídas a drogas anticoncepcionais ${ }^{3,5}$. $\mathrm{Na}$ espécie humana, pioras clínicas e eletrencefalográficas tem sido referidas em pacientes com enxaqueca ${ }^{1,6}$ e com epilepsia ${ }^{2}$. Matsumoto e col. ${ }^{4}$, estudando o registro eletrencefalográfico de 6 pacientes, sem qualquer queixa neuro-psiquiátrica e que faziam uso dessa medicação, encontraram, em todos os casos, uma lentificação do fuso do sono.

\section{MATERIAL, MÉTODO E RESULTADOS}

O material consta de 28 pacientes que estavam tomando medicamento anticoncepcional por via oral por um prazo mínimo de 3 ciclos menstruais consecutivos; nenhuma delas apresentava qualquer manifestação neuropsiquiátrica. Quinze tomaram anticoncepcional do tipo combinado (Lindiol, Ovulen, Primovlar, Anfertil, Anovlar) e 13 do tipo seqüencial (Novulon). Em todas foi realizado um eletrencefalograma em repouso e ativado pela hiperpnéia; em duas pacientes não foi obtido o registro durante o sono. As anormalidades encontradas estão referiảas na tabela 1.

\begin{tabular}{lc}
\hline Tipos de alterações EEG & N.o de casos \\
\hline Irritativa difusa & 4 \\
Irritativa focal & 2 \\
Assimetria da atividade de base & 1 \\
\hline Tabela 1 - Anormalidades eletrencefalográficas assinala- \\
das em 7 das 28 pacientes.
\end{tabular}

Em nenhum dos 26 casos nos quais conseguimos o registro durante o sono, encontramos qualquer lentificação do fuso próprio desse estado.

Trabalho dos Departamentos de Neurologia-Neurocirurgia e de Toco-Ginecologia da Escola Paulista de Medicina, apresentado no $\mathrm{V}$ Congresso Brasileiro de Neurologia e III Congresso Brasileiro de Eletrencefalografia e Neurofisiologia Clínica (São Paulo 12-15 julho 1972): * Prof. Adjunto de Neurologia; ** Prof. Adjunto de Ginecologia; *** Assistente de Neurologia. 


\section{COM ENTARIOS}

O eletrencefalograma realizado em 28 pacientes que estavam fazendo uso de drogas anticoncepcionais por via oral se mostrou anormal em 7 (25\%). Essas alterações foram predominantemente paroxísticas e difusas; tal cifra está bem acima da encontrada na população geral (em torno de 10\%). Correlacionar ou não essas alteraçōes ao uso do anovulatório será objeto de próximo estudo. Devido a própria natureza de nosso material clínico, constituído de pacientes sem qualquer queixa neurológica ou psiquiátrica, pudemos comparar os nossos achados apenas com os de Matsumoto e col., e, contrariamente ao que afirmam estes autores, não registramos lentificação do fuso do sono.

\section{R E S U M O}

Após comentários sobre a literatura, os autores apresentam o material composto de 28 pacientes sem queixa neurológica ou psiquiátrica, que estavam fazendo uso de anticoncepcional oral, e nas quais foi realizado um eletrencefalograma. Este se mostrou anormal em 7 (25\%). As anormalidades foram predominantemente paroxisticas e difusas. Em nenhum caso foi encontrada lentificação do fuso do sono.

\section{S U M M A R Y}

\section{Eletroencephalogram and anticonceptional drugs.}

An electroencephalogram was made in 28 women who had been using oral anticonceptional for at least three menstrual cycles and had no neurological or psychiatric complaint. The electroencephalogram was abnormal in 7 $(25 \%)$. No slowness of the sleep spindle was found in any of the cases.

REFER ENCIAS

1. ANSARI, A. H.; BOYDE, J. R. \& CENTA, C. J. - Electroencephalographic recording during progestation treatment. Fertility and Sterility 21:873, 1970.

2. GRAUNDENZ, G. \& FICHTNER, C. H. - Anticoncepcionais orais e disrritmia cerebral. Rev. Gynecol, e Obstet. 11:318, 1966.

3. KAWAKAMI, M. \& SAWYER, C. H. - Effects of sex hormones and antifertility steroids on brain thresolds in the rabbit. Endocrinology 80:857, 1967.

4. MATSUMOTO, S.; SATO, I.; ITO, T. \& MATSUOKA, A. - Electroencephalographic changes during long term treatment with oral contraceptives. International J. Fertility 11:195, 1966.

5. SAWYER, C. H. \& KAWAKAMI, M. - Control of Ovulation. Ed. Viller C. A., New York, 1961.

6. WEST, J. \& WEST, E. D. - The electroencephalogram and personality of women with headaches on oral contraceptives. Lancet (London) 1:1180, 1966. 